\title{
IMPACTO DE HERBICIDAS EM IsOLADOS DE Trichoderma spp. ${ }^{1}$
}

\author{
Impact of Herbicides on Strains of Trichoderma spp.
}

\author{
REIS, M.R. ${ }^{2}$, LEÃO, E.U. ${ }^{3}$, SANTOS, G.R.. ${ }^{4}$, SARMENTO-BRUM, R.B.C. ${ }^{5}$, GONÇALVES, C.G. ${ }^{6}$, \\ CARDON, C.H. ${ }^{6}$ e SILVA, D.B. ${ }^{6}$
}

\begin{abstract}
RESUMO - O uso de microrganismos é uma alternativa para o controle de doenças em plantas. Todavia, é prudente verificar a interação desse com os demais métodos de controle empregados em determinada cultura. Dessa forma, objetivou-se avaliar a fungitoxicidade dos herbicidas sobre o crescimento e desenvolvimento dos isolados de Trichoderma spp. Utilizou-se o delineamento inteiramente casualizado, em esquema fatorial 6 x 6 x 4, com quatro repetições. O fator A correspondeu aos herbicidas pendimethalin, clomazone, carfentrazone-ethyl, oxadiazon, thiobencarb + propanil e byspiribac-sodium; o fator B, às doses dos herbicidas 0, 25, 50, 75, 100 e 200\% da dose recomendada; e o fator C, aos isolados de Trichoderma spp. AJAM 18, CE 66, TRI 01 e TRI 02. O ensaio foi realizado em condições in vitro; avaliaram-se o crescimento micelial radial (CMR) e a esporulação dos isolados após aplicação dos herbicidas. Observaram-se diferenças de sensibilidade dos isolados para o mesmo produto testado. O oxadiazon reduziu o CMR dos isolados AJAM 18 e TRI 01 em 66 e 35\%, respectivamente. No entanto, reduziu apenas $16 \%$ do CMR do isolado TRI 02 e não alterou o CMR do isolado CE 66 mesmo em 200\% da dose recomendada. Verificaram-se diferentes efeitos dos produtos em cada isolado. A mistura comercial de thiobencarb+ propanil foi altamente tóxica aos isolados de Trichoderma spp., com reduções em torno de 85\% no CMR e no número de esporos. Por outro lado, o byspiribac-sodium pouco afetou os isolados, apresentando reduções inferiores a $10 \%$ no CMR e na esporulação. O carfentrazone-ethyl e byspiribac-sodium demonstraram ser compativeis com os isolados de Trichoderma spp. estudados.
\end{abstract}

Palavras-chave: antagonista, controle biológico, arroz.

\begin{abstract}
The use of microorganisms is an alternative for the control of plant diseases. However, one should verify its interaction with other methods of control used for a particular crop. The objective of this work was to evaluate the effect of herbicide fungitoxicity on the growth and development of Trichoderma spp. strains. A completely randomized design was used in a factorial scheme $6 \times 6 \times 4$, with four replications. Factor A consisted of the herbicides pendimethalin, clomazone, carfentrazone-ethyl, oxadiazon, thiobencarb + propanyl and byspiribac-sodium; factor $B$ consisted of the herbicides doses $0,25,50,75,100$, and 200\% of the recommended dose; and factor $C$ consisted of the Trichoderma spp. strains AJAM 18, CE 66, TRI 01, and TRI 02. The assay was conducted under in vitro conditions, with radial mycelial growth (RMG) and strain sporulation being evaluated after application of the herbicides. A difference was observed in the sensitivity of the strains for the same product tested. Oxadiazon reduced the RMG of the isolates AJAM 18 and TRI 01 in 66\% and 35\%, respectively. However, it reduced only 16\% of the RMG of the isolate TRI 02, and it did not affect the RMG of the isolate CE 66, even when at 200\% of the recommended dose. Different effects of the products were verified on each isolate. The commercial mixture thiobencarb+propanyl was highly toxic to the isolates with reduction of the RMG and in the number of spores around 85\%. On the other hand, byspiribac-sodium had a little effect on the strains with less than $10 \%$ reduction in the RMG and sporulation. Carfentrazone-ethyl and byspiribacsodium were found to be compatible with the isolates of Trichoderma spp. evaluated.
\end{abstract}

Keywords: antagonist, biological control, rice.

Recebido para publicação em 22.3.2012 e aprovado em 26.8.2012.

2 Professor, Instituto de Ciências Agrárias, Universidade Federal de Viçosa, campus de Rio Paranaíba - ICA/UFV-CRP, $<$ marceloreis@ufv.br>; ${ }^{3}$ Eng-â-Agra ${ }^{\text {. }}$, Doutoranda em Proteção de Plantas - UNESP, Botucatu-SP, ${ }^{4}$ Professor, Doutor em Fitopatologia, Universidade Federal do Tocantins, campus de Gurupi - UFT/Gurupi; ${ }^{5}$ Bióloga, Mestre em Produção Vegetal, UFT/Gurupi; ${ }^{6}$ Graduando do curso de Agronomia, Bolsista de Iniciação Científica, UFT/Gurupi.

Planta Daninha, Viçosa-MG, v. 31, n. 2, p. 419-426, 2013 


\section{INTRODUÇÃO}

A maioria das perdas agrícolas ocorre devido às infestações de pragas e doenças, sendo os métodos de mitigação dessas perdas de extrema importância. Desde a origem da agricultura, a criação desses métodos de controle tem sido um desafio; nessa perspectiva, o controle químico é o principal método utilizado (Diniz et al., 2008). Todavia, apenas o uso de pesticidas não é suficiente para solucionar os graves problemas fitossanitários; se utilizado inadequadamente, pode propiciar o impacto negativo ao ambiente. Dessa forma, o controle biológico constitui uma ferramenta do manejo integrado de pragas e doenças em demanda atual e de alta importância para viabilizar a substituição desses produtos.

O uso de microrganismos é uma alternativa para o controle de fitopatógenos, no qual o controle biológico de patógenos pode ser implantado através da introdução de antagonistas, tanto no solo quanto nos órgãos de propagação, ou através da manipulação do ambiente (Santos, 2008).

Os fungos do gênero Trichoderma spp. são capazes de atuar como agentes de controle de doenças de várias plantas cultivadas, promotores de crescimento e indutores de resistência de plantas a doenças (Mohamed \& Haggag, 2006; Fortes et al., 2007). Algumas linhagens desse gênero vêm recebendo grande atenção da comunidade cientifica, por sua versatilidade de ação, como: antibiose, hiperparasitismo e competição. Apresentam uma diversidade de estratégias de sobrevivência, que os tornam fungos altamente competitivos no ambiente, e mostram boa capacidade de proliferação na rizosfera (Melo, 1996; Resende et al., 2004).

A literatura disponivel demonstra que os fungos desse gênero possuem amplas possibilidades para aplicação, tanto no biocontrole de patógenos foliares quanto no de patógenos radiculares das diversas culturas agrícolas (Perelló et al., 2009; Bomfim et al., 2010; Patekoski \& Pires Zottarelli, 2010).

Entretanto, é importante mencionar que os produtos biológicos diferem dos químicos, pois não podem ser utilizados em locais com condições ambientais e biológicas muito diferentes entre si. Isso porque, por serem organismos vivos, devem sobreviver, colonizar e se multiplicar na planta ou no ambiente para que sejam eficazes. A eficiência dos produtos biológicos é afetada diretamente pelos fatores bióticos locais, como a presença de bactérias antagonísticas de solo (Yedidia et al., 2002), e abióticos, como tipo de solo, umidade, $\mathrm{pH}$, temperatura, metais pesados e resíduos de pesticidas (Lucon, 2009).

Os herbicidas, embora visem ao controle de plantas daninhas, podem afetar organismos não alvos, a exemplo dos fungos antagonistas (Peixoto et al., 2010). As evidências de que os herbicidas podem influenciar no crescimento ou no desenvolvimento de diversos fungos fitopatogênicos ou saprófitos do solo foram verificadas por Rosa et al. (2010). Esses autores observaram forte interferência de glyphosate, halosulfuron e sethoxydim sobre isolados de Rhizoctonia, Ceratocystis, Cryphonectria, Phytophthora, Macrophomina, Sclerotium, Fusarium e Mirothecium.

Em relação aos fungos antagonistas, pouco se sabe da interação destes com os demais métodos de controle, principalmente o químico, devido ao potencial de impacto negativo das moléculas disponiveis. Entre os poucos relatos, constata-se que o trifluralin não afeta o crescimento do antagonista Trichoderma viride, porém o paraquat retarda seu crescimento (Ghannoum et al., 1989).

No Estado do Tocantins, o arroz sob sistema irrigado é de grande importância socioeconômica e apresenta condições climáticas favoráveis à alta agressividade de fungos fitopatogênicos - em especial, o Magnaporthe oryzae agente causal da doença brusone. Existe a possibilidade de uso de Trichoderma para o manejo de diversas doenças nesse sistema de cultivo. Uma vez que o número de herbicidas recomendados para essa cultura é grande (55 produtos comerciais) e que essas moléculas podem afetar a ação do agente de controle biológico, objetivou-se avaliar a fungitoxicidade dos herbicidas sobre o crescimento micelial e a esporulação de isolados de Trichoderma spp.

\section{MATERIAL E MÉTODOS}

O experimento foi realizado em condições de laboratório, no período de agosto a novembro de 2010. 
Utilizaram-se quatro isolados de Trichoderma spp., sendo AJAM 18 e CE 66 provenientes da Universidade Federal de Viçosa, cedidos pelo professor Robert W. Barreto, e os isolados TRI 01 e TRI 02, provenientes da Coleção Micológica do Laboratório de Fitopatologia da Universidade Federal do Tocantins, campus de Gurupi.

Os herbicidas utilizados foram: pendimethalin $\left(3,5 \mathrm{~kg} \mathrm{ha}^{-1}\right)$, clomazone $\left(1 \mathrm{~L} \mathrm{ha}^{-1}\right)$, carfentrazone-ethyl $\left(0,125 \mathrm{~L} \mathrm{ha}^{-1}\right)$, oxadiazon $\left(4 \mathrm{~L} \mathrm{ha}^{-1}\right)$, thiobencarb + propanil $\left(8 \mathrm{~L} \mathrm{ha}^{-1}\right) \mathrm{e}$ byspiribac-sodium $\left(0,125 \mathrm{~L} \mathrm{ha}^{-1}\right)$, recomendados para controle de plantas daninhas no arroz na proporção de 0, 25, 50, 75, 100 e 200\% da dose recomendada.

O delineamento utilizado foi inteiramente casualizado, em esquema fatorial $6 \times 6 \times 4$, com quatro repetições, em que o fator $A$ correspondeu aos herbicidas; o fator $\mathrm{B}$, às doses dos herbicidas; e o fator $\mathrm{C}$, aos isolados de Trichoderma spp.

As soluções de herbicidas foram preparadas com $10 \mathrm{~mL}$ de água destilada. As soluções foram esterilizadas por filtragem em disco de filtro de membrana $0,45 \mu \mathrm{m}$, acondicionadas em tubos Falcon previamente autoclavados, e armazenadas em geladeira.

Os isolados foram multiplicados em placas de Petri com meio de cultura BDA (BatataDextrose-Ágar) e incubados a $27{ }^{\circ} \mathrm{C}$ por sete dias. Para instalação dos ensaios, utilizou-se o meio de cultura BDA comercial (Acumedia). Para cada litro de água destilada, adicionaramse $40 \mathrm{~g}$ do meio e $250 \mathrm{mg}$ de antibiótico (Ampicilina). O meio de cultura foi submetido ao processo de esterilização por autoclavagem e foi vertido em placas de Petri de $90 \mathrm{~mm}$ sob condições assépticas.

Em câmara de fluxo laminar, foram espalhados $100 \mu \mathrm{L}$ da solução de cada herbicida por toda a superficie do meio de cultura, com auxílio de uma alça de Drigalski. Em seguida, inoculou-se um disco de micélio de $5 \mathrm{~mm}$ no centro da placa. Após a inoculação, as placas foram incubadas em câmara tipo BOD no escuro, com temperatura de $\pm 27^{\circ} \mathrm{C}$.

O crescimento micelial radial foi acompanhado diariamente, medindo-se o diâmetro da colônia do fungo com um paquímetro digital.
A medição foi feita até a testemunha ocupar toda a superfície do meio de cultura.

Para avaliar o efeito sobre a esporulação, foi realizada, aos sete dias após a inoculação, a quantificação do número de esporos. Para isso, cada placa foi lavada com $10 \mathrm{~mL}$ de água destilada estéril e, com auxílio de um pincel de cerdas macias, realizou-se o desprendimento dos esporos do meio de cultura. A solução foi filtrada em gaze, e a contagem dos esporos, feita em câmara de Neubauer.

Os dados foram submetidos à análise de variância, utilizando-se o teste $F$, e a comparação das médias do fator qualitativo foi feita pelo teste de Tukey a $5 \%$ de probabilidade. Para o fator quantitativo, utilizou-se análise de regressão a $5 \%$ de probabilidade, com a escolha dos modelos baseada na significância dos coeficientes de regressão, no coeficiente de determinação e no fenômeno biológico. Utilizou-se o programa estatístico ASSISTAT (Silva \& Azevedo, 2009).

\section{RESULTADOS E DISCUSSÃO}

Os herbicidas afetaram o crescimento micelial radial (CMR) dos isolados de Trichoderma spp. TRI 01, TRI 02, AJAM 18 e CE 66. Entretanto, não houve ajuste de modelos lineares para essa variável. No campo, o antagonista Trichoderma spp. presente no solo ou na planta pode estar exposto a baixas e altas concentrações dos pesticidas; dessa forma, justifica-se a variação de dosagem dos herbicidas neste trabalho.

O isolado AJAM 18 apresentou-se mais sensivel a partir de $50 \%$ da dose comercial de pendimethalin, com redução de $16,6 \%$ no CMR em relação à dose 0 - sem aplicação de produtos. Com 100 e $200 \%$ da dose comercial, observou-se redução de 55 e $60 \%$ no CMR, respectivamente (Figura $1 \mathrm{~A}$ ).

Por outro lado, o isolado CE 66 demonstrou baixa sensibilidade ao pendimethalin, apresentando redução de apenas 5\% no CMR na dose comercial do produto (Figura 1A). Os isolados TRI 01 e TRI 02 apresentaram comportamento semelhante, tendo em média $20 \%$ do CMR inibido nas doses de 75 e 100, com drástica inibição, 66\%, na dose de 200 (Figura 1A). Resultados contrários foram obtidos por 
Roberti et al. (2006), que não observaram efeitos inibitórios de pendimethalin sobre o CMR de seis espécies de Trichoderma spp.

A interferência do pendimethalin no CMR dos isolados pode ser explicada por ele pertencer ao mecanismo de ação dos inibidores do arranjo dos microtúbulos no processo de mitose, que está presente em todos os seres vivos. De acordo com Shanner \& Weller (2003), esse herbicida pertence ao grupo das dinitroanilinas, que interferem diretamente na divisão celular, impedindo a formação dos microtúbulos responsáveis pela separação dos cromossomos na mitose; consequentemente, ocorrem células multinucleadas - aberrações.

A mistura comercial de thiobencarb+ propanil foi altamente tóxica aos isolados de Trichoderma spp. avaliados. Com apenas 25\% da dose comercial, todos os isolados foram inibidos, com redução média de 40\% no CMR em relação à testemunha sem herbicida (Figura 1B). Na dose comercial, todos os isolados apresentaram reduções drásticas no CRM: em torno de $85 \%$. Com o dobro da dose comercial (200), observou-se mais de $90 \%$ de redução no CRM de todos os isolados (Figura 1B).

Grande parte dos efeitos da mistura é atribuída ao thiobencarb, visto que o propanil, pertencente ao grupo químico das triazinas, é inibidor do fotossistema II da fotossintese e que os fungos não apresentam esse mecanismo de fototrofia. Colla et al. (2008) relataram baixa toxicidade das triazinas aos isolados de Trichoderma spp. e potencial para biorremediação de solos contaminados com triazinas. Apesar do mecanismo de ação pouco conhecido, o thiobencarb - grupo químico dos tiocarbamatos - é inibidor da biossintese de lipídios, proteínas, isoprenoides e flavonoides. Em plantas, têm sido demonstrados efeitos de altas doses de thiobencarb no processo de divisão celular (Silva et al., 2007). Resultados obtidos por Casale \& Hart (1986) mostram o efeito inibitório do thiobencarb sobre o fungo Sclerotinia sclerotiorum.

O oxadiazon não afetou o CMR do isolado CE 66 mesmo na presença do dobro da dose comercial (Figura 1C). O isolado TRI 02 apresentou sensibilidade moderada, com redução de $16 \%$ do CMR na dose comercial, apesar de ter sido sensivel mesmo em baixas doses do produto, em relação aos demais isolados (Figura 1C). Na dose comercial de oxadiazon, os isolados AJAM 118 e TRI 01 foram mais sensiveis, com reduções de 66 e 35\%, respectivamente (Figura $1 \mathrm{C}$ ).

O carfentrazone-ethyl demonstrou pouca toxicidade aos isolados (máximo de 10\% de redução do $\mathrm{CMR}$ ), com exceção do isolado $\mathrm{CE}$ 66, que não apresentou inibição do $C M R$ mesmo em doses elevadas (Figura 1D).

O oxadiazon e carfentrazone-ethyl pertencem aos grupos químicos dos difeniléteres e triazolinonas, respectivamente. Ambos os herbicidas são inibidores da enzima PPO (protoporfirinogênio oxidase), relacionada com a sintese do pigmento clorofila (Silva et al., 2007). Embora o oxadiazon e carfentrazoneethyl tenham o mesmo mecanismo de ação e os fungos não apresentem mecanismos de fototrofia, observou-se intoxicação severa do oxadiazon no isolado AJAM 18 e leve do carfentrazone-ethyl nos isolados TRI $01 \mathrm{e}$ TRI 02. Segundo Malkones (2000), os aditivos presentes na formulação dos pesticidas podem afetar os microrganismos e, em certos casos, até modificar o efeito do pesticida.

Os isolados TRI 01 e TRI 02 não foram sensiveis ao clomazone até 75 e $100 \%$ da dose comercial, respectivamente (Figura $1 \mathrm{E}$ ). Mesmo em doses superiores, ambos os isolados apresentaram intoxicações leves, com redução do CMR menor que $15 \%$. Por sua vez, os isolados AJAM18 e CE 66 foram sensiveis mesmo na menor dose de clomazone, porém as intoxicações foram moderadas, com reduções não excedendo a 30\% do CMR mesmo em doses superiores (Figura 1E).

$\mathrm{O}$ byspiribac-sodium não interferiu no $\mathrm{CMR}$ dos isolados CE66 e AJAM18. Os demais isolados apresentaram no máximo $10 \%$ de redução do CMR, com exceção do TRI 01, que, em contato com o dobro da dose, mostrou redução de 16\% do CMR (Figura 1F). Resultados semelhantes foram obtidos por Rosa et al. (2010), os quais não constataram efeitos de halosulfuron sobre fungos dos gêneros Rhizoctonia, Ceratocystis, Cryphonectria, Phytophthora, Macrophomina, Sclerotium, Fusarium e Mirothecium. Esses autores atribuíram os resultados ao mecanismo de ação do halosulfuron - inibidor da enzima acetolactato sintase (ALS). 
Os inibidores de ALS, inclusive o byspiribacsodium, atuam na rota de sintese dos aminoácidos e em poucas horas bloqueiam a divisão celular e sintese de DNA, levando a planta à morte (Silva et al., 2007).
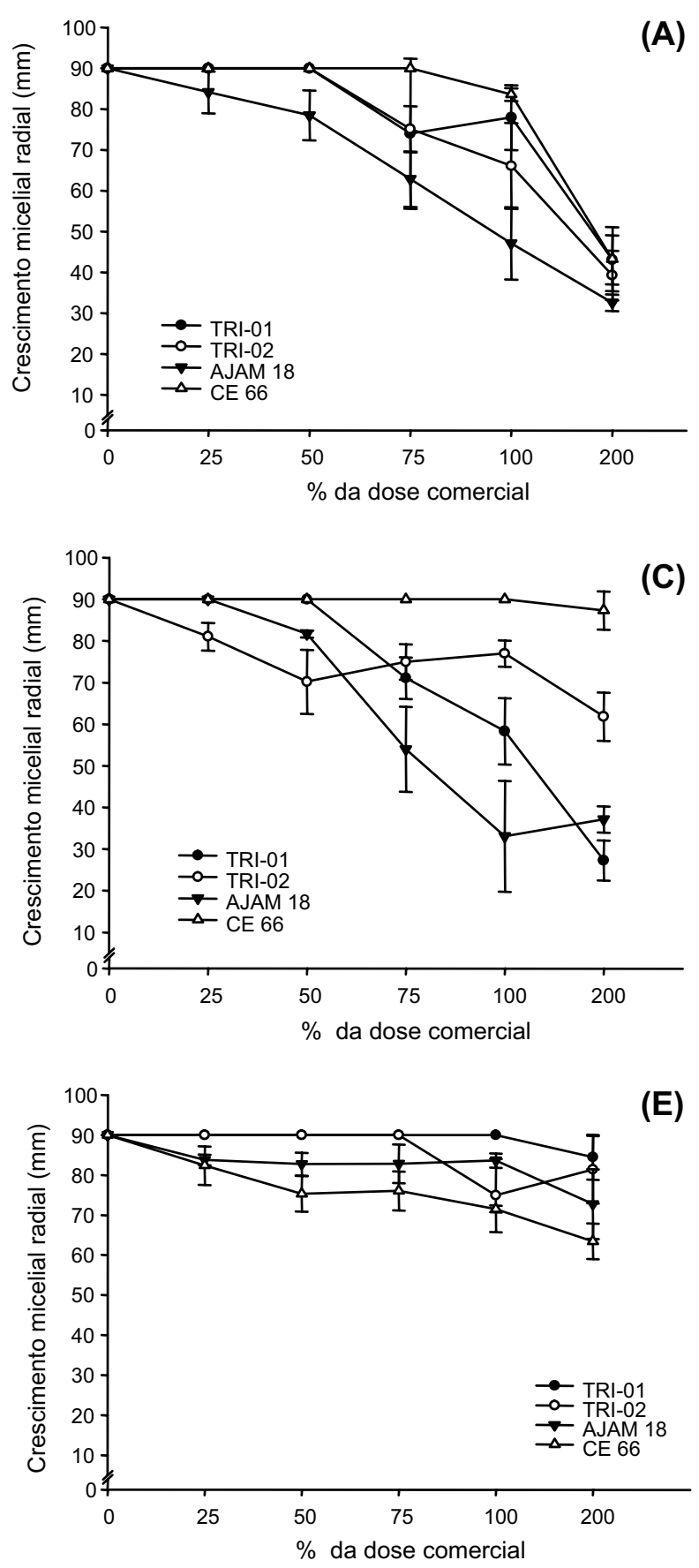

A esporulação dos isolados de Trichoderma spp. na presença de herbicidas foi menos afetada em relação à variável crescimento micelial radial. Observa-se que a esporulação do isolado CE 66 foi inferior à dos demais
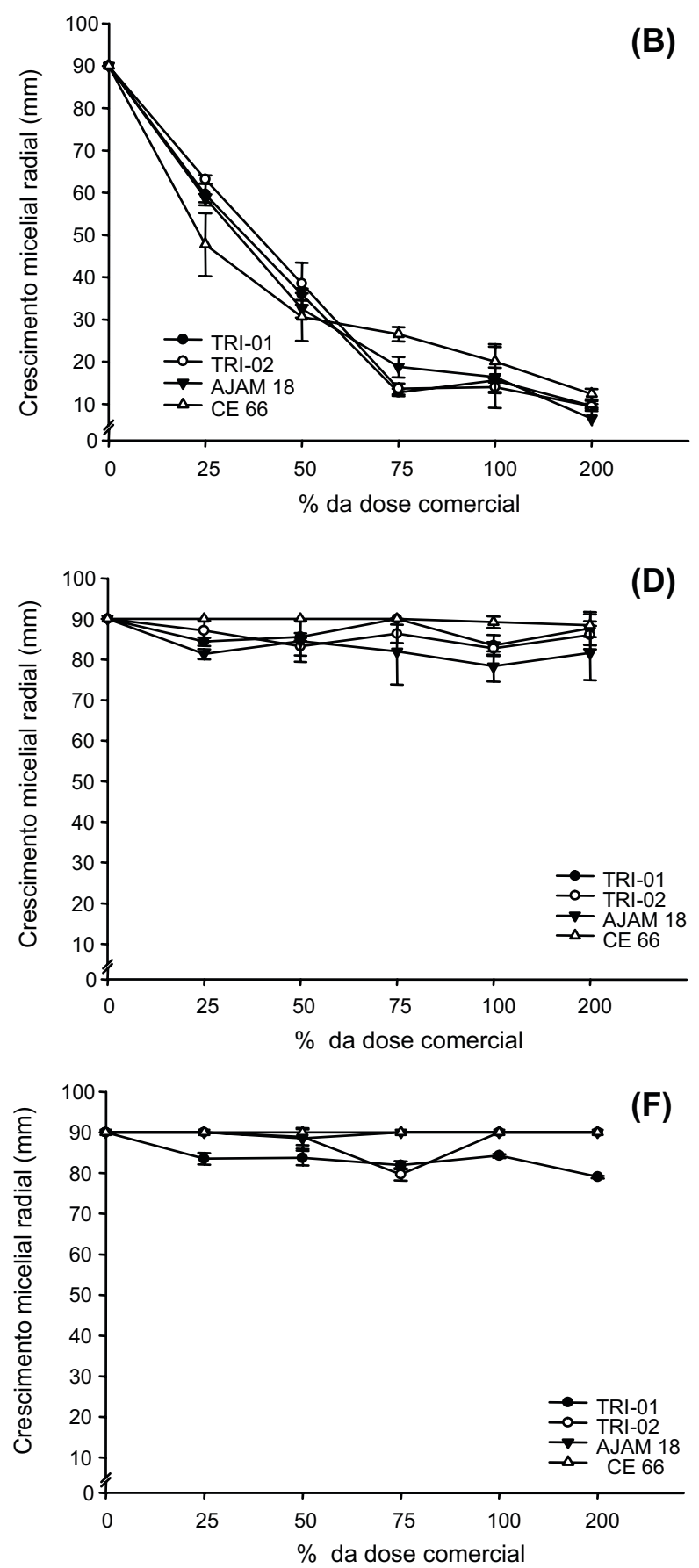

Figura 1 - Crescimento micelial radial $(\mathrm{mm})$ de isolados de Trichoderma spp. com aplicação de herbicidas. Na sequência: (A) pendimethalin, (B) thiobencarb + propanil, (C) oxadiazon, (D) carfentrazone-ethyl, (E) clomazone e (F) byspiribac-sodium Gurupi-TO, 2011. 
isolados mesmo na dose zero, o que demonstra baixa capacidade de produção de inóculo (Tabela 1).

Os dados de esporulação em função de doses de herbicidas ajustaram-se aos modelos lineares de primeiro, segundo e terceiro graus. Para alguns herbicidas, ainda foi verificado o efeito estimulatório em baixas doses (Tabela 1).

Os isolados TRI 01 e 02 apresentaram redução de esporulação na presença de carfentrazone-ethyl com 50 e $75 \%$ da dose recomendada; no entanto, em doses superiores houve recuperação da esporulação, ajustando-se ao modelo quadrático (Tabela 1). Os isolados AJAM 18 e CE 66 apresentaram oscilação, isto é, redução e acréscimo da esporulação no intervalo estudado. Ambos ajustaram-se ao modelo linear cúbico (Tabela 1).

$\mathrm{O}$ pendimethalin afetou negativamente a esporulação do isolado TRI 01 , com redução de 36,8 e $35 \%$ para 75 e $200 \%$ da dose comercial, respectivamente (Tabela 1), sendo ajustado o modelo linear de terceiro grau. Por outro lado, o isolado TRI 02 foi estimulado com 25 e $50 \%$ da dose comercial do produto (Tabela 1). De acordo com Campbell \& Altman (1977), frequentemente, baixas doses de herbicidas têm mostrado efeitos estimulatórios, porém eles podem apresentar efeitos inibitórios

Tabela 1 - Número médio de esporos de isolados de Trichoderma spp. aos sete dias após inoculação e aplicação de diferentes doses de herbicidas. Gurupi-TO, 2011

\begin{tabular}{|c|c|c|c|c|c|c|c|c|c|}
\hline \multirow{2}{*}{ Isolado } & \multicolumn{6}{|c|}{ Dose } & \multirow{2}{*}{ Equação } & \multirow{2}{*}{$\mathrm{R}^{2}$} & \multirow{2}{*}{ Significância } \\
\hline & 0 & 25 & 50 & 75 & 100 & 200 & & & \\
\hline \multicolumn{10}{|c|}{ Carfentrazone-ethyl } \\
\hline TRI-01 & $19,1 a^{*}$ & $14,3 a b$ & $15,7 \mathrm{a}$ & $14,9 \mathrm{a}$ & $15,3 \mathrm{ab}$ & $24,1 \mathrm{a}$ & $y=20,36-0,11 x+0,0008 x^{2}$ & 0,92 & $\mathrm{P}<0,0001$ \\
\hline TR1-02 & $21,5 \mathrm{a}$ & $20,7 \mathrm{a}$ & $14,4 \mathrm{a}$ & $13,3 \mathrm{a}$ & $15,3 \mathrm{ab}$ & $15,5 \mathrm{ab}$ & $y=28,18-0,26 x+0,001 x^{2}$ & 0,90 & $\mathrm{P}<0,0001$ \\
\hline AJAM 18 & $21,6 a$ & $21,5 \mathrm{a}$ & $13,5 \mathrm{a}$ & $18,0 \mathrm{a}$ & $27,2 \mathrm{a}$ & $20,5 \mathrm{a}$ & $y=25,34-0,46 x+0,006 x^{2}-0,00002 x^{3}$ & 0,48 & $\mathrm{P}<0,02$ \\
\hline CE 66 & $8,9 \mathrm{a}$ & $5,0 \mathrm{~b}$ & $20,9 \mathrm{a}$ & $8,0 \mathrm{a}$ & $13,0 \mathrm{~b}$ & $2,7 \mathrm{~b}$ & $y=6,32-0,25 x-0,0043 x^{2}+0,00001 x^{3}$ & 0,54 & $\mathrm{P}<0,01$ \\
\hline \multicolumn{10}{|c|}{ Pendimethalin } \\
\hline TRI-01 & $17,9 \mathrm{a}$ & $14,9 \mathrm{~b}$ & $14,9 \mathrm{a}$ & $11,3 \mathrm{ab}$ & $15,0 \mathrm{a}$ & $11,6 \mathrm{a}$ & $y=17,53-0,22 x-0,0029 x^{2}+0,000009 x^{3}$ & 0,52 & $\mathrm{P}<0,03$ \\
\hline TR1-02 & $12,7 \mathrm{~b}$ & $21,1 \mathrm{a}$ & $16,6 \mathrm{a}$ & $10,3 b$ & $13,8 \mathrm{a}$ & $8,2 \mathrm{a}$ & $y=15,46-0,03 x+0,000017 x^{2}$ & 0,55 & $\mathrm{P}<0,05$ \\
\hline AJAM 18 & $18,0 \mathrm{a}$ & $17,5 \mathrm{ab}$ & $16,0 \mathrm{a}$ & $15,1 \mathrm{a}$ & $12,2 \mathrm{ab}$ & $9,4 \mathrm{a}$ & $y=18,19-0,045 x$ & 0,90 & $\mathrm{P}<0,001$ \\
\hline CE 66 & $8,0 \mathrm{c}$ & $6,7 \mathrm{c}$ & $9,2 \mathrm{~b}$ & $3,6 \mathrm{c}$ & $7,7 \mathrm{~b}$ & $0,9 \mathrm{~b}$ & $y=7,44+0,0033 x-0,0002 x^{2}$ & 0,73 & $\mathrm{P}<0,0001$ \\
\hline \multicolumn{10}{|c|}{ Thiobencarb+Propanil } \\
\hline TRI-01 & $16,1 \mathrm{a}$ & $8,9 \mathrm{bc}$ & $12,8 \mathrm{a}$ & $2,8 \mathrm{a}$ & $4,7 \mathrm{a}$ & $2,1 \mathrm{a}$ & $\mathrm{y}=12,45-0,06 \mathrm{x}$ & 0,65 & $\mathrm{P}<0,0001$ \\
\hline TR1-02 & $4,8 \mathrm{~b}$ & $20,2 \mathrm{a}$ & $6,2 b$ & $0,2 \mathrm{a}$ & $0,6 \mathrm{a}$ & $0,3 \mathrm{a}$ & $y=6,24-0,07 x+0,0002 x^{2}$ & 0,64 & $\mathrm{P}<0,004$ \\
\hline AJAM 18 & $2,1 \mathrm{~b}$ & $8,3 \mathrm{c}$ & $5,7 \mathrm{~b}$ & $3,1 \mathrm{a}$ & $4,7 \mathrm{a}$ & $0,7 \mathrm{a}$ & $\mathrm{y}=4,34+0,028 \mathrm{x}-0,002 \mathrm{x}^{2}$ & 0,59 & $\mathrm{P}<0,04$ \\
\hline CE 66 & $1,1 \mathrm{~b}$ & $13,1 \mathrm{~b}$ & $6,9 \mathrm{~b}$ & $2,7 \mathrm{a}$ & $3,2 \mathrm{a}$ & $1,0 \mathrm{a}$ & $\mathrm{y}=3,11+0,38 \mathrm{x}-0,0062 \mathrm{x}^{2}$ & 0,57 & $\mathrm{P}<0,005$ \\
\hline \multicolumn{10}{|c|}{ Oxadiazon } \\
\hline TRI-01 & $25,8 \mathrm{a}$ & $24,5 \mathrm{a}$ & $20,4 \mathrm{a}$ & $22,1 \mathrm{a}$ & $13,5 \mathrm{a}$ & $10,8 \mathrm{a}$ & $y=25,48-0,08 x$ & 0,77 & $\mathrm{P}<0,0001$ \\
\hline TR1-02 & $14,6 \mathrm{~b}$ & $13,2 \mathrm{~b}$ & $7,0 \mathrm{~b}$ & $10,7 \mathrm{~b}$ & $9,0 \mathrm{a}$ & $8,2 \mathrm{a}$ & $y=10,53-0,06 x+0,0002 x^{2}$ & 0,48 & $\mathrm{P}<0,007$ \\
\hline AJAM 18 & $17,2 \mathrm{~b}$ & $16,0 \mathrm{~b}$ & $11,3 b$ & $13,5 b$ & $12,5 \mathrm{a}$ & $9,9 \mathrm{a}$ & $y=15,94-0,03 x$ & 0,63 & $\mathrm{P}<0,0001$ \\
\hline CE 66 & $6,8 \mathrm{c}$ & $6,7 \mathrm{c}$ & $7,7 \mathrm{~b}$ & $12,5 \mathrm{~b}$ & $11,0 \mathrm{a}$ & $6,1 \mathrm{a}$ & $\mathrm{y}=5,73+0,09 \mathrm{x}-0,0004 \mathrm{x}^{2}$ & 0,63 & $\mathrm{P}<0,0006$ \\
\hline \multicolumn{10}{|c|}{ Clomazone } \\
\hline TRI-01 & $7,0 \mathrm{c}$ & $12,6 \mathrm{~b}$ & $18,4 \mathrm{a}$ & $19,5 \mathrm{a}$ & $15,4 \mathrm{ab}$ & $15,7 \mathrm{~b}$ & $y=8,45+0,18 x+0,0007 x^{2}$ & 0,62 & $\mathrm{P}<0,007$ \\
\hline TR1-02 & $18,7 \mathrm{~b}$ & $18,2 \mathrm{ab}$ & $14,5 \mathrm{a}$ & $16,9 \mathrm{a}$ & $11,9 \mathrm{~b}$ & $11,2 \mathrm{~b}$ & $\mathrm{y}=20,24-0,028 \mathrm{x}$ & 0,50 & $\mathrm{P}<0,001$ \\
\hline AJAM 18 & $27,9 \mathrm{a}$ & $24,2 \mathrm{a}$ & $23,0 \mathrm{a}$ & $23,5 \mathrm{a}$ & $22,9 \mathrm{a}$ & $24,4 \mathrm{a}$ & $y=26,56-0,07 x+0,0003 x^{2}$ & 0,63 & $\mathrm{P}<0,0001$ \\
\hline CE 66 & $3,6 \mathrm{c}$ & $1,7 \mathrm{c}$ & $1,8 \mathrm{~b}$ & $1,9 \mathrm{~b}$ & $1,0 \mathrm{c}$ & $0,9 \mathrm{c}$ & $\mathrm{y}=5,73+0,09 \mathrm{x}-0,0004 \mathrm{x}^{2}$ & 0,28 & $\mathrm{P}<0,02$ \\
\hline \multicolumn{10}{|c|}{ Bispyribac-sodium } \\
\hline TRI-01 & $19,6 \mathrm{a}$ & $21,4 \mathrm{a}$ & $22,1 \mathrm{ab}$ & $16,5 \mathrm{ab}$ & $16,2 \mathrm{~b}$ & $15,3 b$ & $y=21,45-0,036 x$ & 0,67 & $\mathrm{P}<0,0001$ \\
\hline TR1-02 & $17,0 \mathrm{a}$ & $18,5 \mathrm{ab}$ & $24,2 \mathrm{a}$ & $13,5 b$ & $22,3 \mathrm{a}$ & $22,9 \mathrm{a}$ & $y=15,53+0,37 x+0,0058 x^{2}+0,000006 x^{3}$ & 0,56 & $\mathrm{P}<0,001$ \\
\hline AJAM 18 & $17,3 \mathrm{a}$ & $16,7 \mathrm{~b}$ & $19,8 \mathrm{~b}$ & $18,2 \mathrm{a}$ & $20,1 \mathrm{ab}$ & $18,1 \mathrm{~b}$ & $y=18,33-0,02 x+0,0007 x^{2}-0,000002 x^{3}$ & 0,49 & $\mathrm{P}<0,02$ \\
\hline CE 66 & $6,3 b$ & $5,3 \mathrm{c}$ & $4,1 \mathrm{c}$ & $6,8 \mathrm{c}$ & $3,7 \mathrm{c}$ & $3,6 \mathrm{c}$ & $y=6,09-0,018 x+0,00005 x^{2}$ & 0,56 & $\mathrm{P}<0,05$ \\
\hline
\end{tabular}

* Médias seguidas pela mesma letra na coluna não diferem entre si pelo teste de Tukey a $5 \%$ de probabilidade. 
quando usados em altas doses. Para o isolado CE 66, houve estímulo na dose de 50 e $100 \%$ e redução de $88 \%$ na dose de $200 \%$, ajustando-se ao modelo cúbico. A esporulação do isolado AJAM 18 foi reduzida em 37 e 16\% para as doses de 50 e $75 \%$, respectivamente. Contudo, nas doses de 100 e 200\% não foi observada redução, ajustando-se ao modelo quadrático.

A mistura thiobencarb+propanil reduziu drasticamente (70 a 90\%) a produção de esporos dos isolados TRI 01 e 02 a partir de $75 \%$ da dose comercial. Os isolados AJAM 18 e CE 66 foram estimulados a produzir esporos 25 e $50 \%$ da dose comercial. A esporulação de ambos os isolados foi inibida a partir de $75 \%$ da dose (Tabela 1).

Verificou-se que, na presença de oxadiazon, à medida que aumentou a dose, diminuiu linearmente a esporulação dos isolados TRI 01 e AJAM 18. Para os isolados TRI 02 e CE 66 não houve resposta linear; ambos ajustaram-se ao modelo quadrático com mínimo (52\% de redução) em 50\% e máximo (182\% de acréscimo) em $75 \%$ da dose, respectivamente (Tabela 1).

Observou-se efeito estimulatório do clomazone na esporulação do isolado TRI 01 nas doses de 50 e $75 \%$ e do byspiribac-sodium, inibidor da ALS, no isolado TRI 02 com 50, 100 e $200 \%$ da dose comercial (Tabela 1). De modo contrário, Botelho \& Monteiro (2011) observaram redução na esporulação de Beauveria bassiana e Metharizium anisopliae após aplicação do imazapyr - inibidor da ALS.

Os testes in vitro permitem a máxima exposição do microrganismo-teste ao pesticida, porém, no agroecossistema, é pouco provável que a dose de campo atinja totalmente o antagonista, pois, em geral, menos da metade do produto aplicado atinge o alvo. Entretanto, foram detectados efeitos de herbicidas, por exemplo, com apenas $25 \%$ da dose recomendada de thiobencarb + propanil, com redução de quase $50 \%$ do crescimento micelial radial.

Embora, de acordo com Botelho \& Monteiro (2011), os mecanismos de inibição de microrganismos pelos herbicidas sejam ainda pouco conhecidos, neste e em outros trabalhos foram constatados efeitos adversos de pesticidas aos organismos não alvo. Ressalta-se que é necessário que haja integração e averiguação da compatibilidade dos métodos de controle de doenças, pragas e plantas daninhas para que se possa realizar um manejo integrado eficiente e pouco impactante ao ambiente.

Dessa forma, observou-se que os isolados de Trichoderma spp. respondem de forma diferenciada ao mesmo herbicida. Herbicidas com mesmo mecanismo de ação afetam os isolados de forma distinta. Constatou-se também que a mistura thiobencarb + propanil é altamente tóxica para os isolados de Trichoderma spp. estudados.

Os herbicidas carfentrazone-ethyl e byspiribac-sodium são potenciais herbicidas compativeis com os isolados de Trichoderma spp. estudados, podendo ser empregados no manejo integrado da cultura do arroz.

\section{AGRADECIMENTOS}

Ao CNPq, pelo auxílio financeiro, e ao professor Robert Weingart Barreto, da Universidade Federal de Viçosa, por ter concedido dois isolados de Trichoderma spp. utilizados neste estudo.

\section{LITERATURA CITADA}

BOMFIM, M. P. et al. Avaliação antagônica in vitro e in vivo de Trichoderma spp. a Rhizopus stolonifer em maracujazeiro amarelo. Summa Phytopathol., v. 36, n. 1, p. 61-67, 2010.

BOTELHO, A. A. A.; MONTEIRO, A. C. Sensibilidade de fungos entomopatogênicos a agroquímicos usados no manejo da cana-de-açúcar. Bragantia, v. 70, n. 2, p. 361-369, 2011.

CAMPBELL C. L.; ALTMAN, J. Pesticide-plant disease interactions: Effect of cycloate on growth of Rhizoctonia solani. Phytopathology, v. 67, n. 4, p. 557-560, 1976.

CASALE, W. L.; HART, L. P. Influence of four herbicides on carpogenic germination and apothecium development of Sclerotinia sclerotiorum. Phytopathology, v. 76, n. 10, p. 980-984, 1986.

COLLA, L. M. et al . Isolamento e seleção de fungos para biorremediação a partir de solo contaminado com herbicidas triazínicos. Ci. Agrotecnol., v. 32, n. 3, p. 809-813, 2008.

DINIZ, S. P. S. S. et al. Controle do fungo Myrothecium verrucaria por óleos essenciais. R. Bras. Plantas Medic., v. 6, n. 1, p. $60-62,2008$

Planta Daninha, Viçosa-MG, v. 31, n. 2, p. 419-426, 2013 
FORTES, F. O. et al. Promoção de enraizamento de microestacas de um clone de Eucalyptus sp. por Trichoderma spp. R. Árvore, v. 31, n. 2, p. 221-228, 2007.

GHANNOUM, M. A. et al. Variation in growth and fatty acid contents of Trichoderma viride induced by herbicides J. Environ. Sci. Health, v. 24, n.8, p. 957-966, 1989.

LUCON, C. M. M. Promoção de crescimento de plantas com o uso de Trichoderma spp. São Paulo: Instituto Biológico/Centro de Pesquisa e Desenvolvimento de Sanidade Vegetal, 2009. 8 p. (Comunicado Técnico, 94).

MALKONES, H. P. Comparison of the effects of differently formulated herbicides on soil microbial activities - a review. J. Plant Dis. Protec., v. 8, n. 5, p. 781-789, 2000.

MELO, I. S. Trichoderma e Gliocladium como bioprotetores de plantas. Revisão. An. Patol. Plantas, v. 4, n. 1, p. 261-295, 1996.

MOHAMED, H. A. L. A.; HAGGAG, W. M. Biocontrol potential of salinity tolerant mutants of Trichoderma harzianum against Fusarium oxysporum. Braz. J. Microbiol., v. 37, n. 2, p. 181-191, 2006.

PATEKOSKI, K. S.; PIRES ZOTTARELLI, C. L. A Patogenicidade de Pythium aphanidermatum a alface cultivada em hidroponia e seu biocontrole com Trichoderma. Pesq. Agropec. Bras., v. 45, n. 8, p. 805-810, 2010

PEIXOTO, M. F. S. P. et al. Ação do trifluralin na micorrização e crescimento de plantas de amendoim (Arachis hypogaea). Planta Daninha, v. 28, n. 3, p. 609-614, 2010.

PERELLÓ, A. E. et al. Biological control of Septoria tritici blotch on wheat by Trichoderma spp. under field conditions in Argentina. BioControl, v. 54, n. 1, p. 113-122, 2009.
RESENDE, M. L. et al. Inoculação de sementes de milho utilizando o Trichoderma harzianum como promotor de crescimento. Ci. Agrotecnol., v. 28, n. 4, p. 793-798, 2004

ROBERTI, R. et al. Sensitivity of Clonostachys rosea and Trichoderma spp. as potential biocontrol agents to pesticides. J. Phytopathol., v. 154, p. 100-109, 2006.

ROSA, D. D. et al. Efeito de herbicidas sobre agentes fitopatogênicos. Acta Sci. Agron., v. 32, n. 3, p. 379-383, 2010 .

SANTOS, H. A. Trichoderma spp. como promotores de crescimento em plantas e como antagonistas a Fusarium oxysporum. 2008. 111 f. Dissertação (Mestrado em Agronomia) - Universidade de Brasília, Brasilia, 2008.

SILVA, F. A. S. E.; AZEVEDO, C. A. V. Principal components analysis in the Software Assistat-Statistical Attendance. In: WORLD CONGRESS ON COMPUTERS IN AGRICULTURE, 7., 2009, Reno. Anais... Reno: American Society of Agricultural and Biological Engineers, 2009. p. 294-298

SILVA, A. A. et al. Métodos de controle de plantas daninhas. In: SILVA, A. A.; SILVA, J. F. (Eds.). Tópicos em manejo de plantas daninhas. Viçosa, MG: Universidade Federal de Viçosa, 2007. 367 p.

SHANNER, D.; WELLER, S. Cell growth disrupters and inhibitors. In: Herbicide action course. West Lafayette: Purdue University, 2003. p. 255-260.

YEDIDIA, I. et al. Concomitant induction of systemic resistance to Pseudomonas syringae pv. lachrymans in cucumber by Trichoderma asperellum (T-203) and the accumulation of phytoalexins. Appl. Environ. Microbiol., v. 97, n. 12 , p. $567-576,2002$. 